SINAI Journal of Applied Sciences 9 (3) $2020 \quad 285-292$

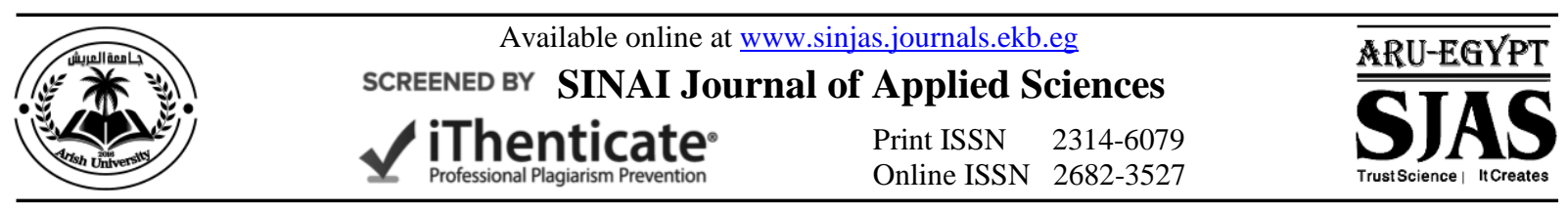

\title{
ANALYTICAL STUDY OF NATURAL AND AGRICULTURAL RESOURCES AFFECTING EDUCATIONAL SPENDING IN JAPAN
}

\author{
Ehab G. Attia ${ }^{* 1}$ and Ali A. Ibrahim²
}

1- PostGrad. Std., Inst. Asian Studies and Res., Zagazig Univ., Egypt.

2- Dept. Agric. Econ., Fac. Agric., Zagazig Univ., Egypt.

ARTICLE INFO

Article history:

Received: 23/09/2020

Revised: 08/07/2020

Accepted: 12/09/2020

Available online: $12 / 10 / 2020$

\section{Keywords:}

Natural and agricultural resources, spending on education, revenues, optimization, value added.

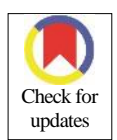

\begin{abstract}
Japan's natural resources are insufficient, especially agricultural lands whose area is not sufficient for the population's need for food, in addition to being a country suffering from natural disasters, and despite this, Japan's economy has reached a global position. Using the estimate of the gradual linear regression equation, it is clear that an increase in mineral resources revenues by one billion dollars leads to an increase in education spending by 1007 million dollars, and it is evident from the increase in the added value of agriculture in Japan by one billion dollars, which leads to an increase in education spending by 1.89 billion dollars. An increase in the total value of agricultural output by one billion dollars leads to a decrease in spending on education by 13 million dollars, while it was found that increasing the agricultural land's contribution from the land area by $1 \%$ leads to a decrease in the agreement on education by 65.4 billion dollars, as it turns out The increase in the number of students in elementary school in Japan by one million pupils leads to an increase in the agreement on education by 400 thousand dollars. It is also clear from the equation that increasing the number of students in technical education in Japan by one million pupils leads to an increase in education spending by 100 thousand dollars, while increasing the number of teachers in secondary education by one million teachers leads to an increase in education spending by one million dollars.
\end{abstract}

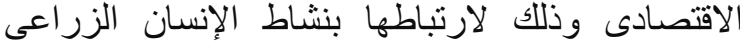

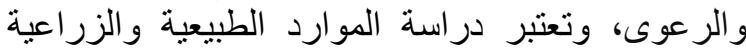

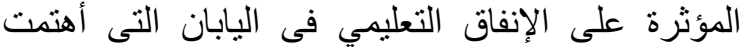

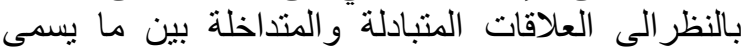
بالعناصر الاقتصادية و غير الاقتصادية. مشكتة البحث

على الرغم من قلة الموارد الطبيعية بدولة اليابان إلا

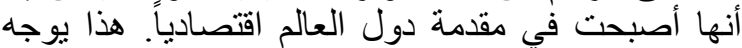

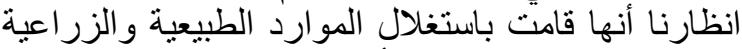

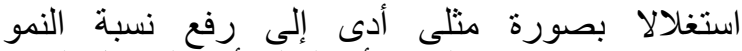

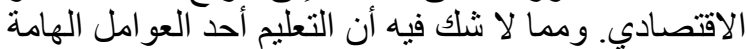

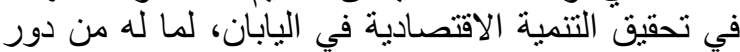

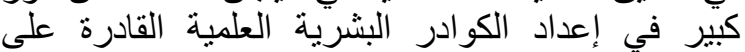

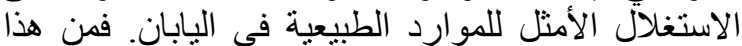
المنطلق تتمثل مشكلة هذه الدراسة في تحليل الإنيل العلاقة بين بين

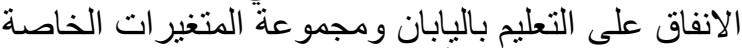

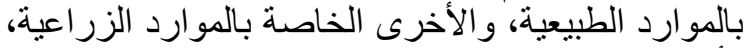
و أيضنا المتغير ات المرتبطة بالتعليم في اليابان.

\section{مقدمة}

تعتبر الموارد الطبيعية فى اليابان غير كافية وخاصة

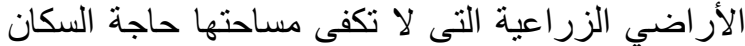

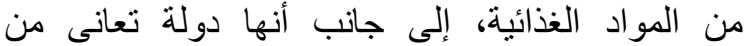

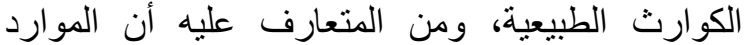

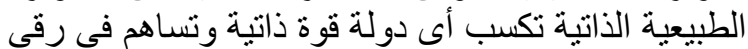

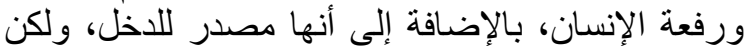

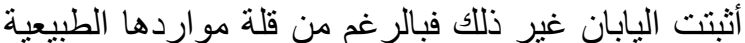

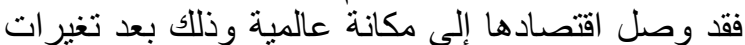

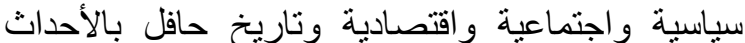

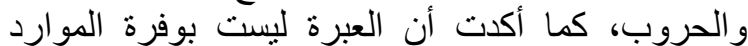

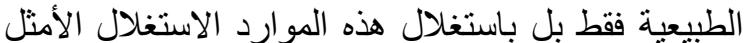

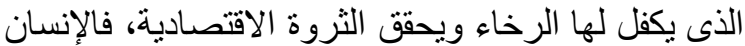

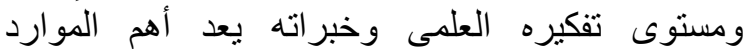

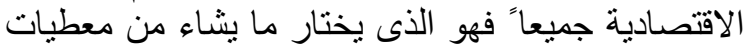

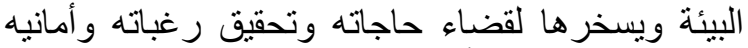
وتعتبر التربة مورد أرضى طبيعى هام تؤثر فى الانتاج

\footnotetext{
* Corresponding author: E-mail address: doaagna2004@gmail.com https://doi.org/10.21608/SINJAS.2020.43883.1004

(C) 2020 SINAI Journal of Applied Sciences. Published by Fac. Environ. Agric. Sci., Arish Univ. All rights reserved.
} 
أهم المتغير ات الخاصة بالمو ارد الطبيعية:

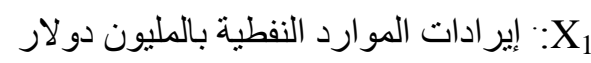
إير ادات موارد الغاز الطبيعي بالمليون دولار : X

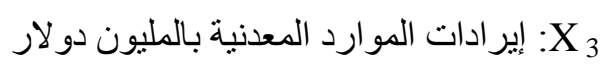

X X

إير ادات موارد الفحم بالمليون دو لار :X

ن 6 الناتج المحلي.

تقدير العلاقة الخطية الانحدار بين المتغير التابع إجمالي

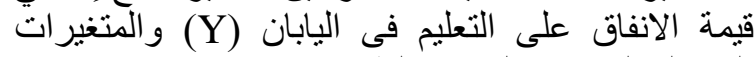
المستقلة الخاصة بالمو ارد الطبيعية :

$$
\mathrm{Y}=142.47+0.1007 \mathrm{X}_{3}
$$

$$
\mathrm{F}=9.6^{* *} \quad \mathrm{R}^{2}=0.26
$$

تبين من تقدير معادلة الانحدار الخطى التدريجى الإبى

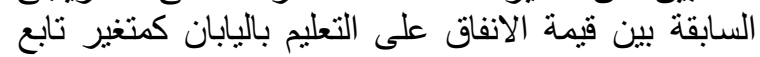

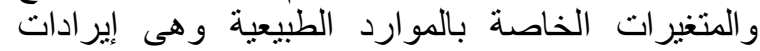

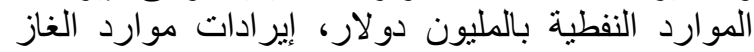

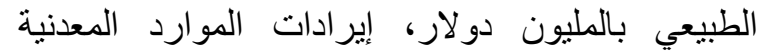

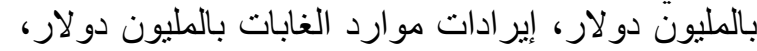

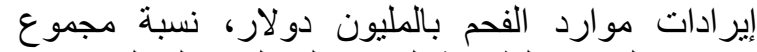
إير ادات الموارد الطبيعية الى إجمالي الناتج المحلي.

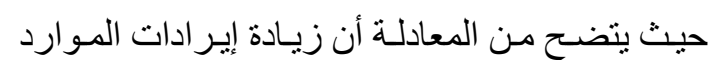

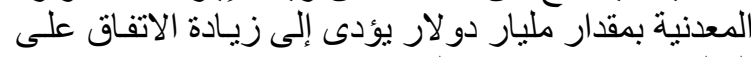

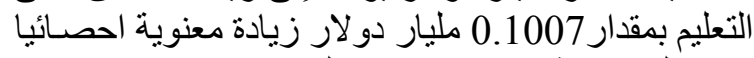
حيث بلغت قيمة اختبار (ت) حو الى 3.09.

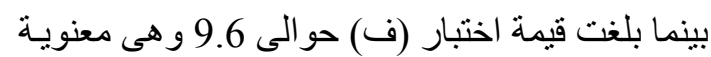

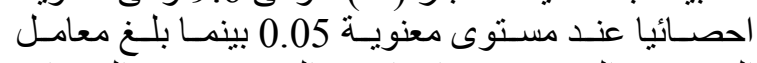

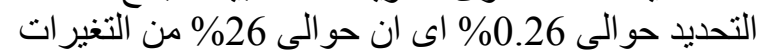

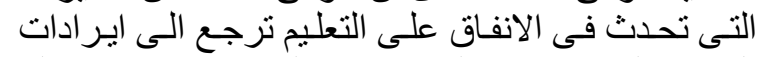

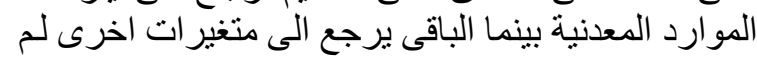
تؤخذ فى الحسبان.

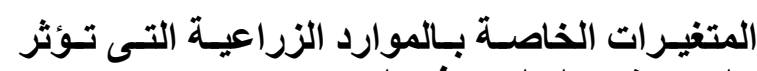
على الانفاق التعليمى فى اليابان

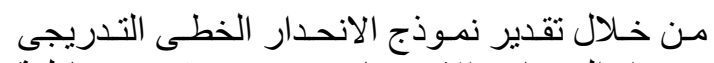

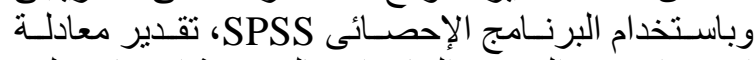

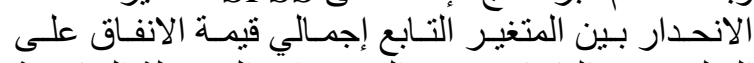

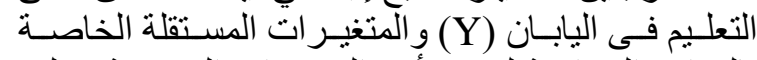

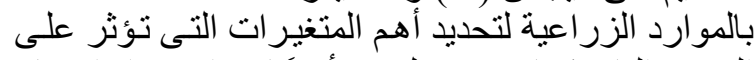

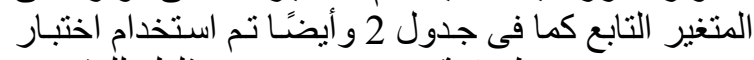

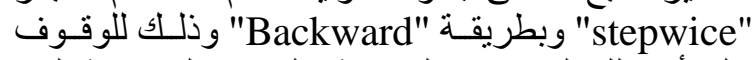

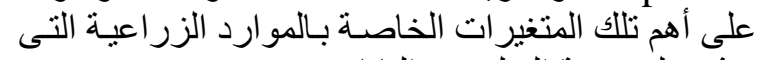
تؤثر على تتمية التعليم فى اليابان.
هدف البحث

يهدف البحث الى دراسة العلاقة بين الموارد الطبيعية والزراعية والإنفاق التعليمي فى اليابان من خلال دراسة العة الطية

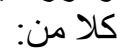

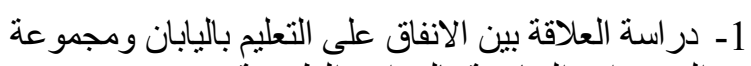
المتغير ات الخاصة بالمو ارد الطبيعية.

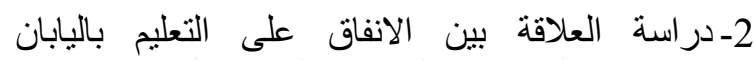
ومجمو عة المتغير اتلات الخاصة بالمو ارد الزر العية.

3-دراسة العلاقة بين الانفاق على التعليم باليابان ومجمو عة المتغير ات الخاصة بالتعليم.

\section{الطريقة البحثية ومصادر البيانات}

اعتمد البحث على أساليب التحليل الوصفي والكمي

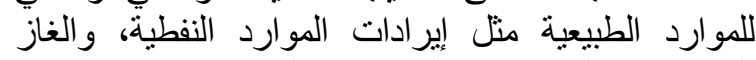

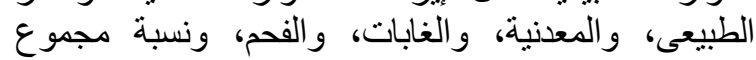

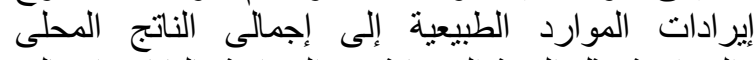

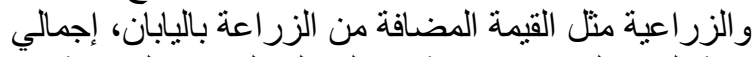

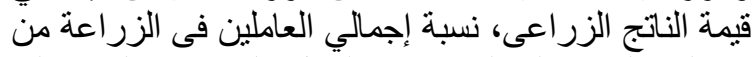

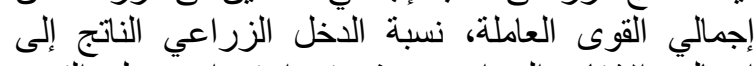

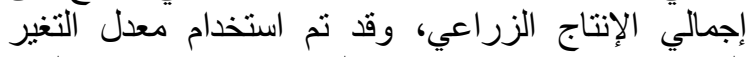

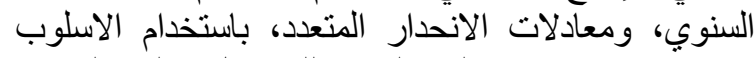

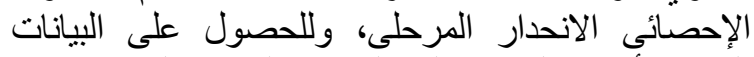

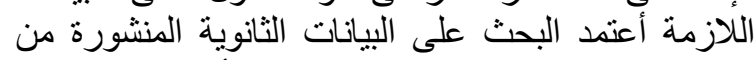
موقع البنك الدولى وموقع منظمة البنى الأغذية والزية الزراعة

.(FAO)

\section{النتائج و المناقثة}

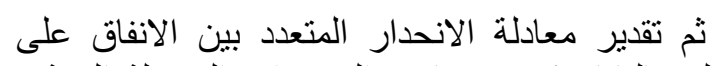

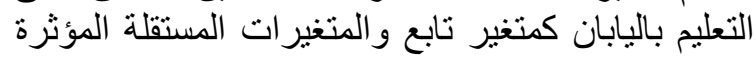
خلال الفترة (1990- 2018) فى الصورة الخير الخطية كما يلى: $Y_{X}=a_{0}+a_{1} X_{1}+a_{2} X_{2}+a_{3} X_{3}+a_{4} X_{4+} a_{5} X_{5+} a_{6} X_{6} \ldots . .+a_{n} X_{n}$ وقد تم تقسيم المتغير ات الى متغير ات خاصة بالمئ والموارد

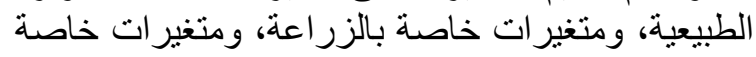
بالتعليم.

المتغيرات الخاصة بالموارد الطبيعية

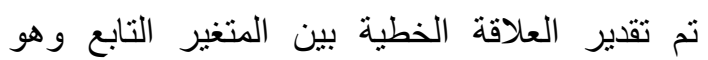

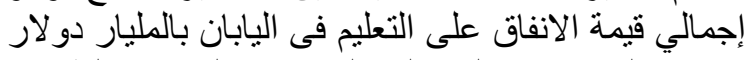

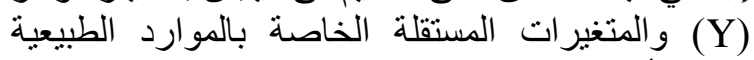

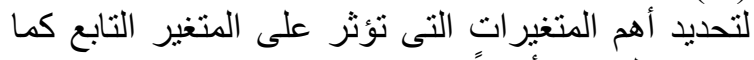

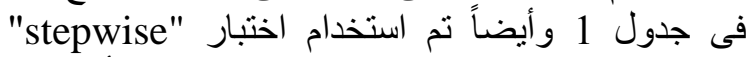

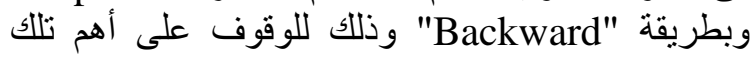
المتغيرات التى تؤثر على الانفاق على التعليم فى اليابان. 
جدول 1. مجموعة المتغيرات الخاصة بالموارد الطبيعية التى تؤثر على الانفاق التعليمى فى اليابان خلال الفترة (1990-

(2018

\begin{tabular}{|c|c|c|c|c|c|c|c|}
\hline$\times 6$ & $\mathrm{x} 5$ & $\mathrm{x4}$ & $\mathbf{x} 3$ & $\mathbf{X} 2$ & $\mathbf{x 1}$ & $\mathbf{y}$ & السنوات \\
\hline 0.036 & 41.0 & 848.9 & 91.9 & 56.8 & 82.5 & 129.0 & 1990 \\
\hline 0.027 & 31.9 & 794.6 & 26.3 & 41.0 & 57.3 & 147.0 & 1991 \\
\hline 0.022 & 13.3 & 722.5 & 18.7 & 29.4 & 72.0 & 140.4 & 1992 \\
\hline 0.021 & 0.9 & 799.2 & 14.6 & 36.2 & 71.6 & 153.3 & 1993 \\
\hline 0.017 & 1.0 & 685.5 & 25.8 & 45.1 & 62.9 & 178.2 & 1994 \\
\hline 0.015 & 11.4 & 693.0 & 13.6 & 48.2 & 71.6 & 192.5 & 1995 \\
\hline 0.018 & 2.2 & 691.8 & 14.6 & 59.4 & 84.9 & 160.4 & 1996 \\
\hline 0.015 & 0.7 & 556.0 & 2.1 & 60.8 & 63.6 & 144.8 & 1997 \\
\hline 0.013 & 2.0 & 459.1 & 0.4 & 38.9 & 33.1 & 119.3 & 1998 \\
\hline 0.011 & 0.9 & 390.2 & 0.3 & 50.3 & 51.5 & 137.0 & 1999 \\
\hline 0.012 & 2.3 & 379.6 & 0.6 & 96.7 & 100.1 & 151.5 & 2000 \\
\hline 0.011 & 19.2 & 315.9 & 0.3 & 88.8 & 51.3 & 132.0 & 2001 \\
\hline 0.011 & 2.0 & 317.4 & 6.2 & 89.6 & 47.9 & 127.3 & 2002 \\
\hline 0.012 & 3.1 & 351.0 & 16.1 & 119.5 & 53.6 & 139.4 & 2003 \\
\hline 0.011 & 30.2 & 280.9 & 24.6 & 156.6 & 56.2 & 150.2 & 2004 \\
\hline 0.015 & 19.3 & 379.6 & 32.1 & 183.8 & 93.6 & 145.8 & 2005 \\
\hline 0.019 & 21.3 & 425.2 & 90.6 & 232.1 & 101.3 & 136.5 & 2006 \\
\hline 0.023 & 32.9 & 525.1 & 94.9 & 278.2 & 121.3 & 137.0 & 2007 \\
\hline 0.026 & 86.3 & 622.9 & 105.9 & 344.2 & 132.8 & 151.7 & 2008 \\
\hline 0.021 & 21.8 & 542.4 & 150.1 & 336.1 & 68.2 & 161.1 & 2009 \\
\hline 0.021 & 41.7 & 560.0 & 231.8 & 270.7 & 93.2 & 179.9 & 2010 \\
\hline 0.024 & 68.1 & 564.3 & 325.6 & 351.9 & 142.1 & 196.3 & 2011 \\
\hline 0.023 & 38.7 & 593.1 & 294.0 & 352.4 & 162.4 & 203.7 & 2012 \\
\hline 0.026 & 25.9 & 671.2 & 236.5 & 271.7 & 151.2 & 166.6 & 2013 \\
\hline 0.030 & 23.1 & 761.5 & 195.2 & 296.7 & 184.5 & 149.9 & 2014 \\
\hline 0.030 & 13.8 & 665.5 & 195.0 & 353.5 & 90.2 & 135.4 & 2015 \\
\hline 0.026 & 15.7 & 721.2 & 228.0 & 229.9 & 73.4 & 150.8 & 2016 \\
\hline 0.028 & 21.5 & 735.4 & 232.9 & 270.5 & 99.1 & 148.8 & 2017 \\
\hline 0.029 & 27.4 & 749.6 & 237.7 & 311.2 & 124.8 & 158.4 & 2018 \\
\hline 0.02 & 21.4 & 579.4 & 100.2 & 175.9 & 89.6 & 152.6 & المتوسط \\
\hline 0.01 & 20.4 & 167.7 & 105.8 & 122.1 & 37.7 & 20.8 & الالحعراف \\
\hline 0.01 & 0.7 & 280.9 & 0.3 & 29.4 & 33.1 & 119.3 & الحد الادنى \\
\hline 0.04 & 86.3 & 848.9 & 325.6 & 353.5 & 184.5 & 203.7 & الحدد الاقصى \\
\hline
\end{tabular}


مسـاهمة الأر اضـي الزر اعية من مسـاحة الأر اضـي بينمـا

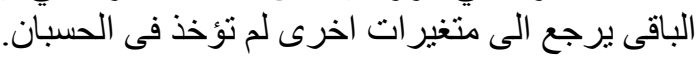

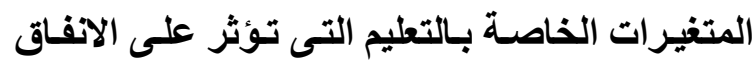
التعليمى فى اليابان

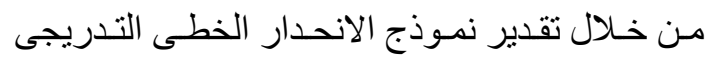

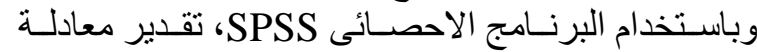
الانحدار بين المتغير التابع و هو إجمالي قيمة الانفاق على لئى

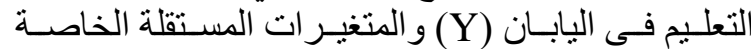

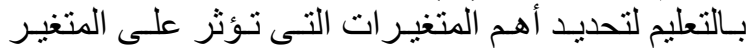

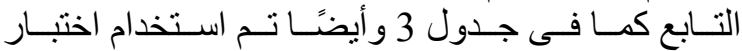

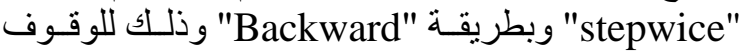

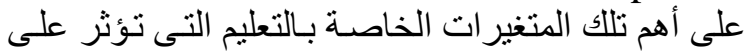
تنمية التعليم فى اليابان.

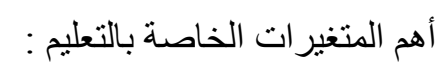

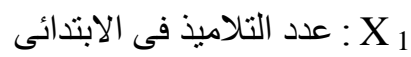

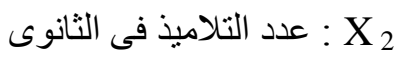
X : X $X_{3}$ عد : عدد المدرسين فى التعليم الابتدائى : : X ع $X_{5}$

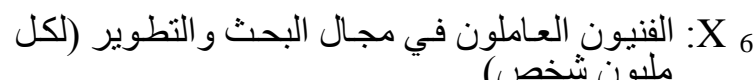
الباحثون العاملون في مجال البحث و التطوير (لكل

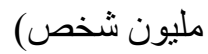

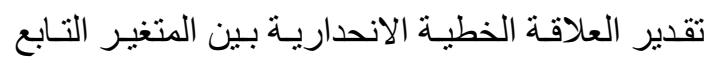

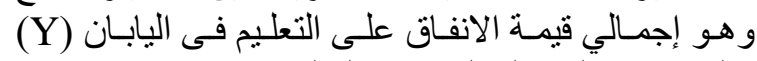

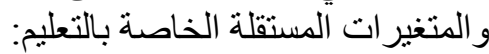

$\mathrm{Y}=-408.4+0.0004 \mathrm{X}_{1}+0.0001 \mathrm{X}_{3}+0.001 \mathrm{X}_{5}$

$$
\mathrm{F}=5.2^{* * * 7)} \quad \mathrm{R}^{2}=0.31
$$

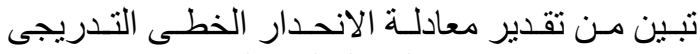

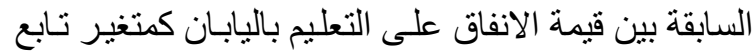

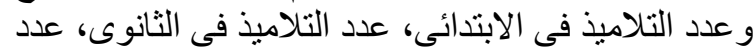

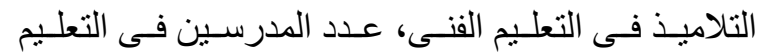

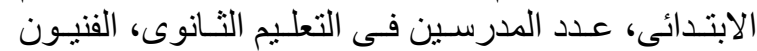

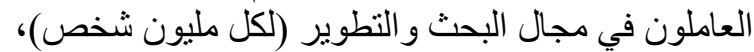
لباحثون العاملون في مجال البحث و التطوير (لكل مليون

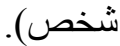

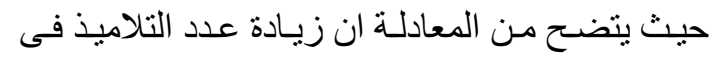

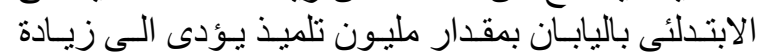

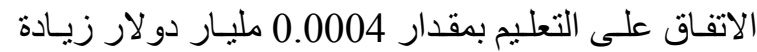

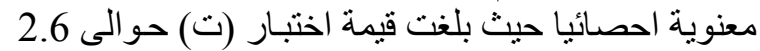
كما يتضح من المعادلة ان زيادة عدد التلاميذ في التعليم
أهم المتغير ات الخاصة بالمو ارد الزر اعية: X X X

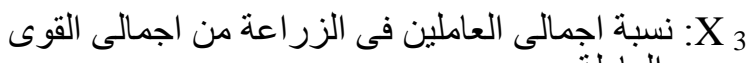

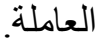

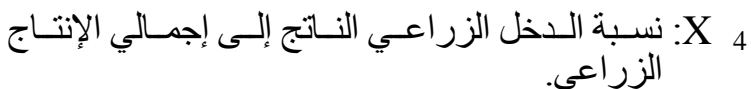
XX

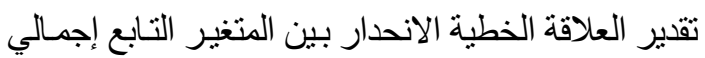

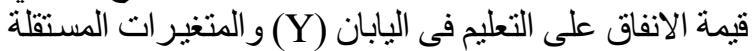

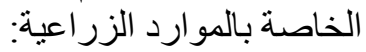

$$
\begin{gathered}
\mathrm{Y}=-203965.7+1.89 \mathrm{X}_{1}-0.013 \mathrm{X}_{2}-65.4 \mathrm{X}_{5} \\
\begin{array}{c}
(3.5)^{*} \\
\mathrm{~F}=6.5^{* *}
\end{array} \quad \mathrm{R}^{2}=0.44
\end{gathered}
$$

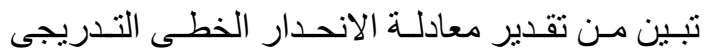

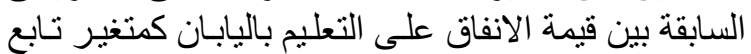

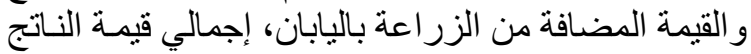

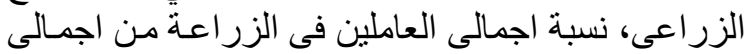

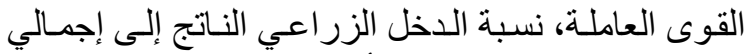

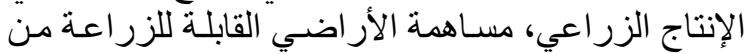

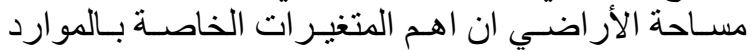

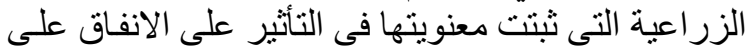

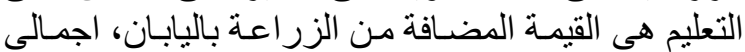

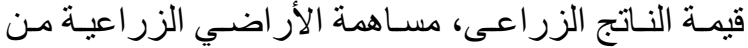
مساحة الأر اضجي.

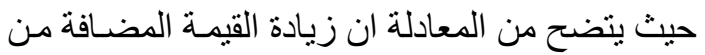

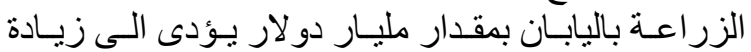

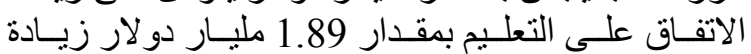

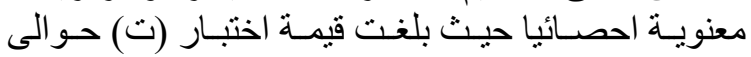

كما يتضـح من المعادلة ان زيـادة اجمالى قيمـة النـاتج

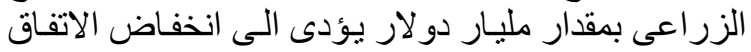

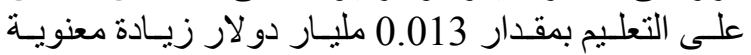

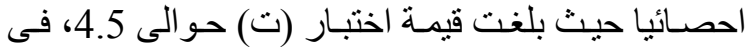

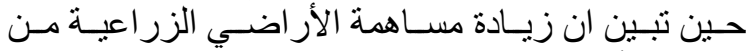

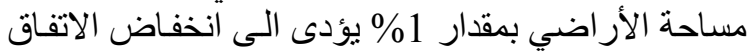

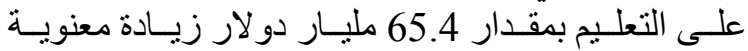

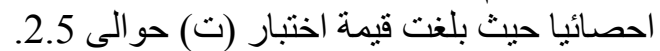
بيتما بلغت قيمة اختبار (ف) حو الى 6.5 و هى معنويـة

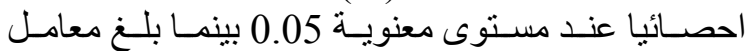

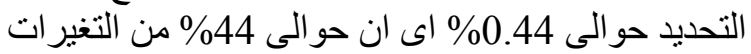

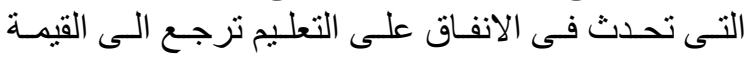

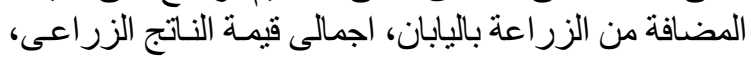




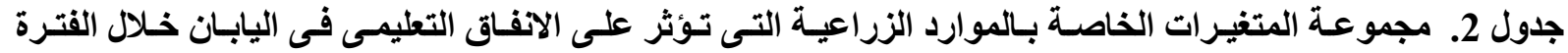

(2018-1990)

\begin{tabular}{|c|c|c|c|c|c|c|}
\hline X5 & $\mathrm{x} 4$ & $\mathbf{x} 3$ & $\mathrm{x} 2$ & $\mathbf{x 1}$ & $\mathbf{y}$ & \\
\hline 15.61 & 377800 & 41.9 & 11493 & 130.1296 & 129 & 1990 \\
\hline 15.51 & 377800 & 44.3 & 10450 & 121.9044 & 146.962 & 1991 \\
\hline 15.4 & 377800 & 31 & 7402 & 113.6792 & 140.398 & 1992 \\
\hline 15.29 & 377800 & 31 & 7473 & 105.454 & 153.34 & 1993 \\
\hline 15.18 & 377800 & 32 & 7544 & 95.287 & 178.2 & 1994 \\
\hline 14.93 & 377800 & 32 & 7615 & 92.093 & 192.5 & 1995 \\
\hline 14.81 & 377800 & 32 & 7686 & 81.929 & 160.389 & 1996 \\
\hline 14.69 & 377800 & 33 & 7757 & 68.751 & 144.756 & 1997 \\
\hline 14.57 & 377800 & 33 & 7829 & 65.832 & 119.26 & 1998 \\
\hline 14.46 & 377800 & 33 & 7900 & 73.018 & 136.997 & 1999 \\
\hline 14.43 & 377800 & 39 & 9130 & 75.072 & 151.496 & 2000 \\
\hline 13.15 & 377880 & 45 & 10360 & 59.501 & 132.04 & 2001 \\
\hline 13.07 & 377890 & 50 & 11591 & 57.287 & 127.339 & 2002 \\
\hline 12.99 & 377900 & 38 & 8466 & 58.452 & 139.398 & 2003 \\
\hline 12.93 & 377910 & 38 & 8445 & 59.82 & 150.185 & 2004 \\
\hline 12.87 & 377910 & 38 & 8512 & 53.516 & 145.811 & 2005 \\
\hline 12.81 & 377920 & 37 & 8332 & 49.576 & 136.541 & 2006 \\
\hline 12.76 & 377930 & 36.6 & 8259 & 47.903 & 136.958 & 2007 \\
\hline 12.7 & 377940 & 37 & 8466 & 53.322 & 151.731 & 2008 \\
\hline 12.64 & 377947 & 36.6 & 8190 & 56.636 & 161.081 & 2009 \\
\hline 12.6 & 377950 & 35 & 8121 & 62.83 & 179.925 & 2010 \\
\hline 12.51 & 377955 & 33.7 & 8246 & 66.218 & 196.317 & 2011 \\
\hline 12.48 & 377960 & 34.7 & 8525 & 70.828 & 203.695 & 2012 \\
\hline 12.45 & 377962 & 34.7 & 8467 & 56.929 & 166.623 & 2013 \\
\hline 12.39 & 377962 & 33.9 & 8364 & 51.233 & 149.941 & 2014 \\
\hline 12.33 & 377971 & 37.4 & 8798 & 48.895 & 135.399 & 2015 \\
\hline 12.26 & 377970 & 40.8 & 9203 & 59.666 & 150.832 & 2016 \\
\hline 12.2 & 377970 & 44.2 & 9608 & 57.797 & 148.766 & 2017 \\
\hline 12.13 & 377969 & 47.6 & 10013 & 55.928 & 158.366 & 2018 \\
\hline 13.5 & 377886.1 & 37.3 & 8698.1 & 70.7 & 152.6 & المتوسط \\
\hline 1.2 & 72.4 & 5.1 & 1126.5 & 22.7 & 20.8 & الاتحراف المعيارى \\
\hline 12.2 & 377800.0 & 31.0 & 7402.0 & 47.9 & 119.3 & الحد الادنى \\
\hline 15.6 & 377971.0 & 50.0 & 11591.0 & 130.1 & 203.7 & الحدد الاقصى \\
\hline
\end{tabular}


جلول 3. مجموعة المتغيرات الخاصة بالتعليم التى تؤثر على الانفاق التعليمى فى اليابان خلال الفترة (1990-2018)

\begin{tabular}{|c|c|c|c|c|c|c|c|c|}
\hline $\mathbf{x} 7$ & x6 & x5 & $\mathrm{x4}$ & $\mathrm{x} 3$ & $\mathrm{x} 2$ & $\mathbf{x 1}$ & $\mathbf{y}$ & السنوات \\
\hline 4196.6 & 606.5 & 651728.0 & 454109.0 & 1450704.0 & 11143930.0 & 9606627.0 & 129.0 & 1990 \\
\hline 4327.1 & 616.7 & 658569.0 & 452849.0 & 1497046.0 & 11025720.0 & 9373295.0 & 147.0 & 1991 \\
\hline 4457.5 & 627.0 & 663215.0 & 453379.0 & 1452097.0 & 10676866.0 & 9157429.0 & 140.4 & 1992 \\
\hline 4588.0 & 637.2 & 695707.0 & 440769.0 & 1483198.0 & 10255337.0 & 8947226.0 & 153.3 & 1993 \\
\hline 4718.5 & 647.4 & 702575.0 & 461729.0 & 1435724.0 & 10202510.0 & 8798082.0 & 178.2 & 1994 \\
\hline 4820.3 & 666.7 & 635646.0 & 464431.0 & 1218987.0 & 9878568.0 & 8612106.0 & 192.5 & 1995 \\
\hline 4874.4 & 662.5 & 629836.0 & 430958.0 & 1170216.0 & 9643000.0 & 8370246.0 & 160.4 & 1996 \\
\hline 4928.5 & 658.3 & 628376.0 & 425714.0 & 1144475.0 & 9602422.0 & 8105629.0 & 144.8 & 1997 \\
\hline 5135.4 & 683.0 & 622207.0 & 426440.0 & 1125375.0 & 9430212.0 & 7855387.0 & 119.3 & 1998 \\
\hline 5174.6 & 663.8 & 619504.0 & 366550.0 & 1091676.0 & 8958699.0 & 7691872.0 & 137.0 & 1999 \\
\hline 5077.6 & 619.1 & 615158.0 & 363880.0 & 1047720.0 & 8782114.0 & 7528907.0 & 151.5 & 2000 \\
\hline 5112.8 & 538.3 & 612629.0 & 362605.0 & 1015005.0 & 8605812.0 & 7394582.0 & 132.0 & 2001 \\
\hline 4871.2 & 524.1 & 610372.0 & 365540.0 & 993848.0 & 8394050.0 & 7325866.0 & 127.3 & 2002 \\
\hline 5093.9 & 526.2 & 609557.0 & 371688.0 & 960517.0 & 8131217.0 & 7268928.0 & 139.4 & 2003 \\
\hline 5098.9 & 570.2 & 607663.0 & 378950.0 & 922184.0 & 7894456.0 & 7257223.0 & 150.2 & 2004 \\
\hline 5303.5 & 558.9 & 607062.0 & 382738.0 & 889381.0 & 7710439.0 & 7231854.0 & 145.8 & 2005 \\
\hline 5332.6 & 575.2 & 609966.0 & 386443.0 & 872418.0 & 7561241.0 & 7229135.0 & 136.5 & 2006 \\
\hline 5325.2 & 583.8 & 613851.0 & 390542.0 & 859374.0 & 7427059.0 & 7220111.0 & 137.0 & 2007 \\
\hline 5108.3 & 587.5 & 617642.0 & 391967.0 & 857049.0 & 7355678.0 & 7166285.0 & 151.7 & 2008 \\
\hline 5098.8 & 581.8 & 624215.0 & 369388.0 & 821213.9 & 7299966.0 & 7156039.0 & 161.1 & 2009 \\
\hline 5103.2 & 582.3 & 631675.0 & 399424.0 & 800564.4 & 7296330.0 & 7098862.0 & 179.9 & 2010 \\
\hline 5109.9 & 559.1 & 634117.0 & 401773.0 & 851048.0 & 7284867.0 & 7029265.0 & 196.3 & 2011 \\
\hline 5032.8 & 512.6 & 638414.0 & 405144.0 & 849773.0 & 7288434.0 & 6923951.0 & 203.7 & 2012 \\
\hline 5147.5 & 513.8 & 637889.0 & 406511.0 & 848498.0 & 7280759.0 & 6801716.0 & 166.6 & 2013 \\
\hline 5328.6 & 537.0 & 640579.0 & 408247.0 & 847223.0 & 7227485.0 & 6714539.0 & 149.9 & 2014 \\
\hline 5173.4 & 522.0 & 642465.0 & 410355.0 & 836605.0 & 7221135.0 & 6638174.0 & 135.4 & 2015 \\
\hline 5210.0 & 502.6 & 644351.0 & 414683.0 & 832882.0 & 7157298.0 & 6581949.0 & 150.8 & 2016 \\
\hline 5304.9 & 520.7 & 646237.0 & 417071.0 & 821023.0 & 7093113.0 & 6531731.0 & 148.8 & 2017 \\
\hline 5399.8 & 538.9 & 648123.0 & 419459.0 & 812494.0 & 7028928.0 & 6481513.0 & 158.4 & 2018 \\
\hline 5015.6 & 583.6 & 634459.6 & 407701.2 & 1027873.0 & 8443367.1 & 7589604.4 & 152.6 & المتوسط \\
\hline 304.8 & 55.3 & 23920.6 & 31483.0 & 234581.3 & 1344087.6 & 901428.0 & 20.8 & الانحراف المعيارى \\
\hline 4196.6 & 502.6 & 607062.0 & 362605.0 & 800564.4 & 7093113.0 & 6531731.0 & 119.3 & الحد الادنى \\
\hline 5399.8 & 683.0 & 702575.0 & 464431.0 & 1497046.0 & 11143930.0 & 9606627.0 & 203.7 & الحدد الاقصى \\
\hline
\end{tabular}


3- يجـب الاسـتفادة بـالموارد الزر اعيـة فـى تتمبـة التعليم

4- يجب الاستفادة بالعو امل التهى تؤدى إلى تتميـة التعليم بالدول النامبة.

\section{المراجع}

رايشـاور، أدويـن (1989). اليابـانبون، ترجمـةن: ليلـى الئى

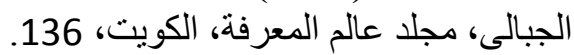

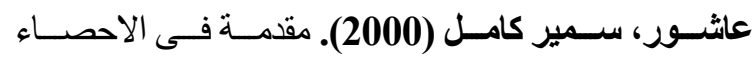

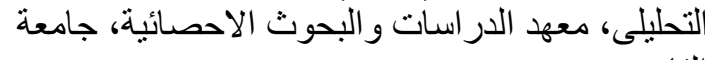
القاهرة.

متاح على الثبكة العنكبوتية WWW.Worldbank البنك الدولى بيانـات غير منشـورة، شبكة الانترنت، اليابـان .2018

هارون، على أحمد (2003). أسس الجغر افيا الاقتصادية، دار الفكر العربى، القاهرة (201).

Abdelmalki, L. (2010). Mundler Patrick, Economie de L'environnement. Developpement Durable, Editions De Boeck Université, Bruxelles.
الفنـى باليابـان بمقدار مليون تلميـذ يؤدى الـى زيـادة

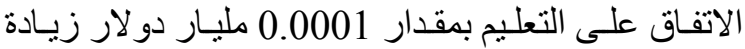

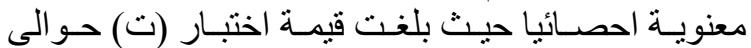

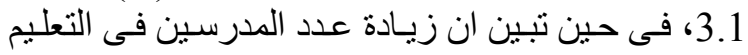

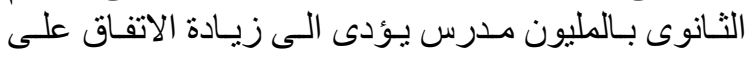
التعليم بمقدار 0,001 مليار دو لار زيـادة معنويـة احصـائيا حيث بلغت قيمة اختبار (ت) حو الى ملى

بيتما بلغت قيمة اختبار (ف) حو الى 5,2 وهى معنوية احصائيا عند مستوى معنوية 0,05 بينما بلغ معار معامل

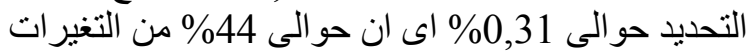

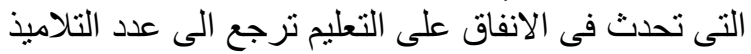

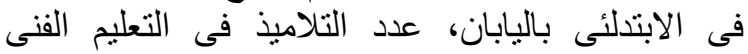

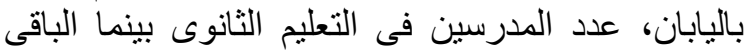
يرجع الى متغير ات اخرى لم تؤخذ فى الحسبان. التوصيات 1- الاسـتفادة مـن اهميـة دور المـوارد الطبيعيـة فـى تنميـة

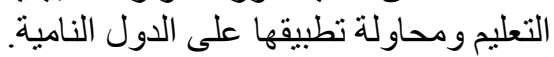

2- يجـب الاسـتفادة بـالموارد الطبيعيـة فـى تنميـة التعلـيم بالدول النامية. 


$$
\begin{aligned}
& \text { المُلخص العربي } \\
& \text { دراسة تحليلية للموارد الطبيعية والزراعية المؤثرة على الإنفاق التعليمي فى اليابان } \\
& \text { إيهاب جمال حسنى عطية1 - على أحمد ابراهيم² }
\end{aligned}
$$

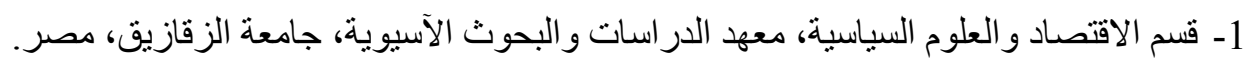

$$
\begin{aligned}
& \text { 2- قسم الاقتصاد الزر اعي، كلية الزر اعة، جامعة الزقازيق، مصر. }
\end{aligned}
$$

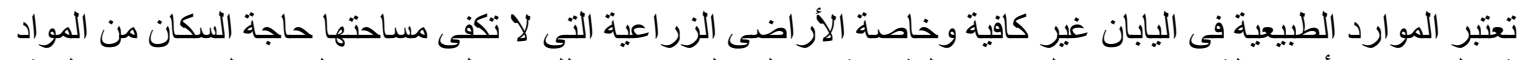

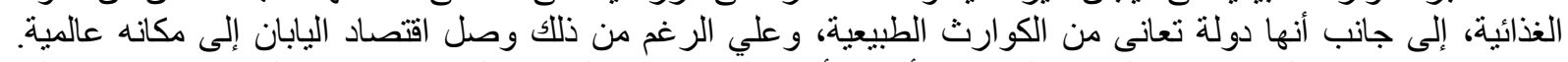

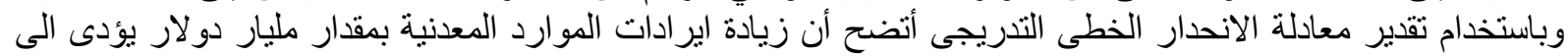

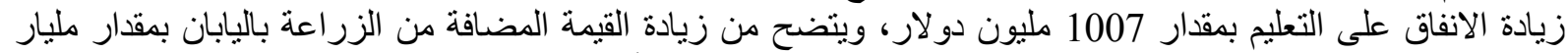

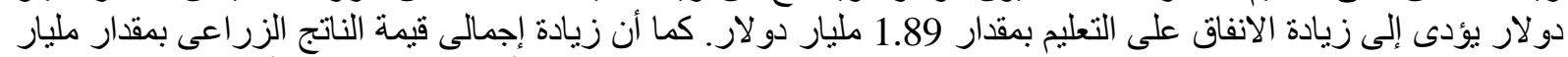

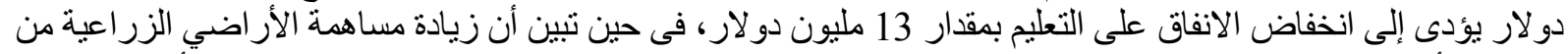

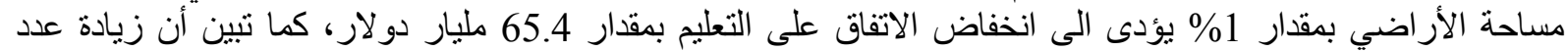

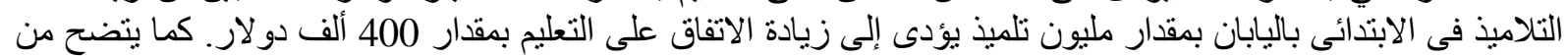

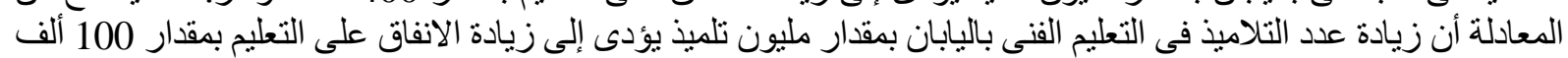

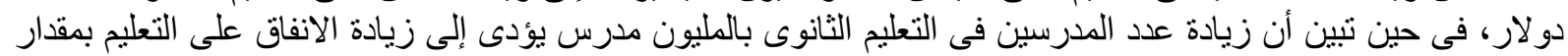
مليون دو لار.

الكلمات الاسترشادية: الموارد الطبيعية والزر اعية، الإنفاق علي التعليم، إير ادات، الاستغلال الأمثل، القيمة المضافة.

أستاذ الاقتصاد الزراعي المتفرغ، كلية الزراعة، جامعة الزقازيقة الزازيق، مصر. أستاذ الاقتصاد الزراعي، كلية الزراعة الزراعة، جامعة الزراعة الزقازيق، مصعة. 